\begin{abstract}
Dr. habil. Nótári, Tamás
Staats- und Rechtswissenschaftliche Fakultät der Károli Gáspár Universität Budapest

Rechtswissenschaftliches Institut des Sozialwissenschaftlichen Forschungszentrums der Ungarischen Akademie der Wissenschaften
\end{abstract}

\title{
Dignitas und otium. \\ Die Wurzeln zweier konservativer Grundwerte im ciceronianischen Staatsdenken
}

Um Cicero zu würdigen bedürfte es einen anderen Cicero, denn wie schon Quintilian in seiner Institutiones oratoriae zutreffend formulierte, ist Cicero kein menschlicher Name, sondern vielmehr der ein Synonym der Beredsamkeit. ${ }^{1}$ Jedoch dürfen wir aus Bewunderung für den Redner und Staatsmann den auf dem Gebiet der Staats- und Rechtphilosophie ebenfalls bleibendes schaffenden Cicero vergessen. Für die ideale Verbindung forensischer Redekunst und staatsphilosophischen Gedankengutes liefert uns seine Sestiana ein Paradebeispiel. In seiner im März 56 v. Chr. gehaltenen Verteidigungsrede für Publius Sestius, der aufgrund der lex Plautia de vi angeklagt war, legte Cicero überzeugend dar, daß es sich beim Verhalten und Handeln seines Klienten um Notwehr handelte. ${ }^{2}$ Der Zeitpunkt der Sestiana war für Cicero die erste Gelegenheit nach seiner Rückkehr aus dem Exil sein politisches Programm der Neuordnung der von discordia heimgesuchten res publica öffentlich zu formulieren. Die Neuordnung heißt in diesem Fall die Wiederherstellung der sullanischen Verfassung - bzw. der von Cicero als sullanisch verstandenen Verfassung - und der Führungsrolle des Senats. Daß der Prozess mit einem Freispruch für Sestius endete war wahrscheinlich nicht ausschließlich der genialen forensischen Taktik Ciceros zu verdanken, sondern auch jener Überzeugungskraft, mit der er die Hörer für sein mit mitreißendem Pathos dargelegtes staatspolitisches Programm begeisterte. ${ }^{3}$

Der vorliegende Beitrag möchte erstens einen kurzen Einblick in die Ereignisse des Exils und der Rückkehr Ciceros, d.h. die politisch-juristischen Hintergründe der Rede geben. Zweitens sollte es dem staatsphilosophischen Gedankengut der Sestiana Aufmerksamkeit gewidmet werden, da Cicero einerseits sich der rhetorischen Situation anpassend, andererseits seiner politischen Überzeugung treubleibend eine einzigartig brilliante Definition der zur Führung des Staates berufenen Optimaten formuliert. Im Zusammenhang mit der Kategorie der Optimaten stellt er die grundlegende Bedeutung zweier, für den optimus quisque im öffentlichen Leben maßgebende Wertbegriffe, dinitas und otium in den Mittelpunkt. Die dignitas als Festhalten an moralischen Werten, an Haltung und Würde und das otium als Kriterium für materiellen Wohlstand (Rechts)Sicherheit und Ruhe im öffentlichen Leben bilden im Gedankengang Ciceros eine harmonische Einheit, eine Synthese des cum dignitate otium.

1. Der historische Hintergrund der Rede für Sestius

Zweifelsohne war sein Konsulatsjahr der Höhepunkt der politischen Karriere des homo novus Cicero, dessen Vorfahren keine kurulischen Ämter innehatten. ${ }^{4}$ Durch das Aufdecken der

\footnotetext{
${ }^{1}$ Quint. inst. 10, 110-112.

${ }^{2}$ Zur lex Plautia siehe Kunkel 1962: 123 f.; Münzer 1920: 200 ff.; Classen 1973: 60 ff.; Stroh 1975: 246 f.; Mommsen 1899: $564 \mathrm{f}$.

${ }^{3}$ Vgl. Meyer 2005: 9 ff., 20 ff.

${ }^{4}$ Hierzu siehe ausführlich Meier 1968: $61 \mathrm{ff}$.
} 
catilinarischen Verschwörung, die Rettung des Staates und das Erschaffen der concordia ordinum stieg er als pater patriae in die Reihe der principes civitatis empor, und hoffte mit gutem Recht auf einen Zeit seines Lebens andauernden Dank seiner Mitbürger und einen ausschlaggebenden Einfluß auf das künftige öffentliche Leben. ${ }^{5}$ Diese Hoffnungen täuschten ihn aber früher, als es sich irgend jemand gedacht hätte: zwei der am 10. Dezember 63 ins Amt tretenden Volkstribunen, L. Calpurnius Bestia und Q. Caecilius Metellus starteten sofort eine heftige Agitation gegen Cicero indem sie ihn beschuldigten, daß er trotz des senatus consultum ultimum, der allgemeinen Zustimmung des Senats und der Öffentlichkeit fünf als hostes rei publicae geltende Catilinarier widerrechtlich hinrichten ließ, und legten Veto dagegen ein, daß er an seinem letzten Amtstag, am 29. Dezember eine Rede an das Volk richtete. ${ }^{6}$ Somit hatte er lediglich die Gelegenheit einen öffentlichen Eid zu leisten, in dem er beschwor einerseits während seines Konsulats Recht und Gesetz geachtet, andererseits den Staat gerettet zu haben. ${ }^{7}$ Schon am Ende des Jahres 61 beklagt er in einem Brief an Atticus, daß weder die von ihm erschaffene concordia, noch seine Verdienste ihm den nötigen Schutz zu geben vermögen. ${ }^{8}$

Diesen Schutz hoffte er bei Pompeius zu finden, der ihn wiederum für die Ziele des Triumvirats zu gewinnen versuchte. Cicero konnte zwar genau sehen, daß sein Beitritt zum Triumvirat ihm den nötigen Schutz vor den Angriffen wegen seines Vorgehens gegen die Catilinarier sichern könnte, wollte sich jedoch dank seiner moralischen Weit- und politischen Kurzsicht nicht mit dem von ihm als gefährlichen Popularen angesehenen Caesar auf einen Plattform stellen. Um stärkeren Druck auf ihn auszuüben fand Caesar ein williges Werkzeug in Ciceros Todfeind P. Clodius Pulcher. ${ }^{9}$

Clodius beschoß, um alle Hindernisse für seine Rache an Cicero aus dem Wege zu räumen, sich zum Volkstribun wählen zu lassen. ${ }^{10}$ Nachdem er mit den Konsulen des Jahres 58, d.h. Gabinius und Caesars Schwiegervater Piso ein Pakt abschloß, daß die beiden nach ihrem Amtsjahr mit entsprechenden finanziellen und militärischen Konditionen die von ihnen gewünschten Provinzen erhalten würden, ${ }^{11}$ ließ er am Ende des Jahres 58 die lex Clodia de capite civium mit rückwirkender Kraft erbringen, die das Hinrichten römischer Bürger ohne ein Gerichtsurteil und der Möglichkeit der provocatio ad populum mit der Todesstrafe bedrohte.

Das Gesetz enthielt zwar nicht ausdrücklich den Namen Ciceros, das Ziel dieses in Gesetzesform gehüllten Racheaktes war jedoch für jeden unmißverständlich. Cicero und Tausende von Bürgern legten Tauerkleidung an und wandten sich an den Senat, während Clodius und seine Banden Unruhe stifteten. Piso blieb der Senatssitzung fern, und Gabinius lehnte es strikt ab Partei für Cicero zu ergreifen. Auf Anraten des Volktsribuns L. Ninius legte der gesamte Senat Trauergewand an. ${ }^{12}$ Daraufhin rief Gabinius die contio plebis zusammen und verkündete, daß der Senat jegliche politische Macht und Bedeutung verloren hätte und drohte den Rittern wegen der von Cicero angeordneten Hinrichtung der fünf Catilinarier am 5. Dezember 63 blutige Rache an. Um seinen Worten mehr Gewicht zu verleihen, schickte er in seinem Edikt den sich für Cicero einsetzenden L. Aelius Lamia ins Exil. ${ }^{13}$ Die Konsulen

\footnotetext{
${ }^{5}$ Materiale 2004: 147; Krüger 1991: 187.

${ }^{6}$ Vgl. Bleicken 1975: 92 ff.; Uttschenko 1978: 121 ff.; Drexler 1976: 124 ff.; Giebel 1977: 45 ff.

${ }^{7}$ Cic. fam. 5, 2, 7.

${ }^{8}$ Cic. Att. 1, 17, 10.

9 Über die Hintergründe der Feindschaft zwischen Cicero und Clodius siehe Rundell 1979: 301 ff.; Heinze 1925: 193 ff.; Spielvogel 1997: 56 ff.; Epstein 1986: 229 ff.; Salzman 1982: 299.; Geffcken 1973.

${ }^{10} \mathrm{Vgl}$. Gruen 1966: 120 ff.; Moreau 1982: $45 \mathrm{ff}$.

${ }^{11}$ Cic. Sest. 24; 33.

12 Cic. Sest. $25 \mathrm{ff}$.

${ }^{13}$ Cic. Sest. 28 ff.
} 
ordneten an, daß der Senat die Trauerkleidung ablegen sollte, ${ }^{14}$ während Clodius verkündete, daß er mit dem Einverständnis und im Sinne der Triumviren handelte. ${ }^{15}$ Öffentlich äußerte sich zwar keiner der Tiumviren, Cicero hoffte jedoch, daß Pompeius seine Verspechen ihm zur Hilfe zu kommen einlösen würde. ${ }^{16}$

Gabinius und Piso äußerten sich dahin, daß die von Cicero anbefohlene Hinrichtungen unrechtmäßig gewesen seien, da er römische Bürger ohne ein Gerichtsurteil und Möglichkeit der provocatio in Anspruch genommen haben zu dürfen erdrosseln ließ, ließen allerdings die ihm vom Senat kraft des senatus consultum ultimum gegebene Vollmacht außer Acht. ${ }^{17}$ Caesar, der seiner Zeit gegen die Hinrichtung der fünf Verschwörer Stellung nahm, ${ }^{18}$ stand der rückwirkenden Anwendung der lex Clodia de capite civium skeptisch gegenüber.

Nachdem er die düsteren Aussichten wahrnahm, zog Cicero im März 58 freiwillig ins Exil, ${ }^{19}$ da - wie er im nachhinein seine Entscheidung zu begründen versuchte - sein Bleiben einen blutigen Bürgerkrieg ausgelöst hätte, was er nicht hätte verantworten können. ${ }^{20}$ Sein Haus wurde geplündert, die Beute teilten sich Clodius und die das Staatswohl für die von ihnen erwünschten Provinzen aufopfernde Konsulen. ${ }^{21}$ Mit einem weiteren Gesetz erreichte Clodius, daß Ciceros gesamtes Vermögen konfisziert werde, und daß dem ins Exil gejagten pater patriae es verwährt bleiben sollte sich in Italien niederlassen zu dürfen. ${ }^{22}$ An den Stätten seiner zerstörten Villa an der Palatin weihte Clodius unter skandalösen Umständen, was später noch Cicero zugute kommen sollte, der Göttin Libertas einen Tempel. ${ }^{23}$

Clodius konnte sich nunmehr als wirklicher Herr Roms fühlen, und versuchte jeglichen Widerstand mit seinen Knüppelbanden auszurotten. ${ }^{24}$ Das Wüten des Clodius brachte als natürliche Folge mit sich, daß die Rückberufung, bzw. Rehabilitierung Ciceros als politische Forderung laut wurde. Der ehemalige Konsul, L. Aurelius Cotta erklärte, daß zur Rückberufung Ciceros ein einfacher Senatsbeschluß reichte, da die bezügliche lex Clodia überhaupt keine Gesetzeskraft besäße. Pompeius forderte einen Volksbeschluß, weil er damit rechnete, daß die Popularen Krawallen veranstalten werden, und der Senat pflichtete seiner Meinung bei. Der Volkstribun Sex. Atilius Serranus forderte als einziger eine Bedenkzeit von zwei Tagen, und verhinderte durch seine Interzession immer wieder eine Entscheidung. ${ }^{25} \mathrm{Da}$ ergriffen die acht zu Cicero loyalen Volkstribune unter der Führung des Q. Fabricius die Initiative und legten der Volksversammlung am 23. Januar einen Entwurf über die Rückberufung des verbannten Konsuls vor. Noch in derselben Nacht besetzten Clodius mit seiner bewaffneten Sklavenhorde und sein Bruder, der Praetor Ap. Claudius Pulcher mit seiner Gladiatorentruppe das Forum. Es kam zu blutigen Auseinandersetzungen, Ciceros Bruder, Quintus kam knapp mit dem Leben davon, Clodius und seine Knüppelbanden verbreiteten in den nächsten Tagen Terror auf Roms Straßen, die Konsulen und der Senat zeigte sich machtlos. $^{26}$

Nachdem der Volkstribun T. Annius Milo vergebens versucht hatte eine Anklage de vi gegen Clodius durchzubringen, beschloß er dem Wüten der clodianischen Banden mit seiner eigenen

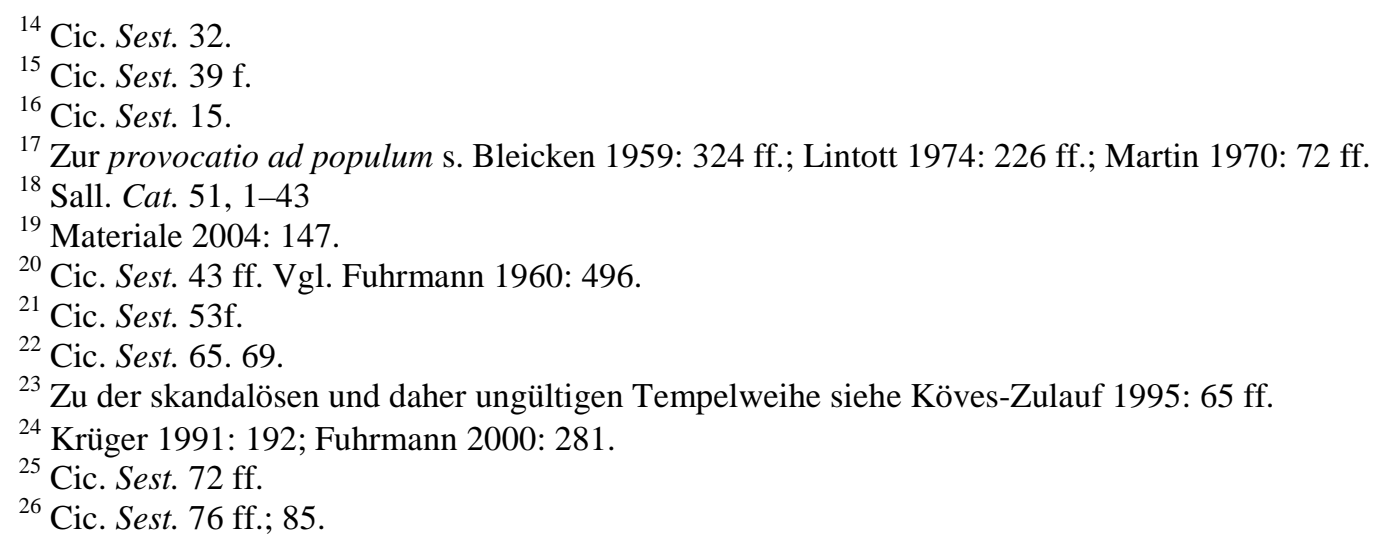


Truppe ein Ende zu setzen. ${ }^{27}$ Dem Beispiel des Milo folgte auch der Volkstribun P. Sestius, nachdem Milo einem Attentat fast zu Opfer gefallen war. ${ }^{28}$ Die von Milo und Sestius aufgestellten Wehrtruppen gewannen in einigen Tagen Oberhand, und es stellte sich in Rom eine verhältnismäßige Ruhe ein. Am 4. August beschloß die Volksversammlung, daß Cicero nach Rom zurückkehren sollte, ${ }^{29}$ dessen Heimkehr sich zu einem wahren Triumphzug gestaltete. $^{30}$

Clodius versuchte allerdings weiterhin Unruhe $\mathrm{zu}$ stiften, machte Cicero in aller Öffentlichkeit für die Inflation verantwortlich und verjagte die von Cicero mit dem Wiederaufbau seines Hauses beauftragten Arbeiter. ${ }^{31}$ Milo gedachte wiederum vor der quasetio de vi Anklage gegen Clodius zu erheben, ließ aber auf Anraten des Senats von seinem Vorhaben ab. ${ }^{32}$ Clodius unterließ es jedoch nicht auch den sich für die Rückberufung Ciceros einsetzenden Volkstribunen P. Sestius zu verfolgen, und erhob gegen ihn Anklage sowohl wegen ambitus, ${ }^{33}$ als auch wegen vis. ${ }^{34}$ Der Prozess wegen vis endete für Sestius am 14. März mit einem Freispruch. ${ }^{35}$

Der Ankläger P. Albinovanus ${ }^{36}$ beschuldigte Sestius für seine politischen Ziele Gladiatoren ausgehoben und bewaffnet zu haben. ${ }^{37}$ Clodius ließ unter anderen L. Aemilius Paulus, Gellius Publicola $^{38}$ und P. Vatinius in den Zeugenstand rufen, ${ }^{39}$ den Vorsitz des Gerichtshofes hatte der Praetor M. Aemilius Scaurus inne. Als Verteidiger traten Q. Hortensius, M. Crassus, L. Licinius Calvus und seiner Gewohnheit gemäß als letzter Cicero selbst auf. ${ }^{40}$ Was die politische Überzeugung der im Prozeß auftretenden Advokaten anbelangt, zeichnete sich ein buntes Bild heraus: zwischen dem Triumvir Crassus, dem konservativen Hortensius und dem eher popular eingestellten Calvus stellte Cicero als politisch zentrale Persönlichkeit das Gleichgewicht her. Es mag unter anderen eben diese Zusammensetzung der Verteidiger Cicero dazu ermutigt haben in einem tiefgehenden, schwungvollen und dennoch pathetischen Exkurs die Prinzipien des römischen Gemeinwesens, bzw. die Rolle und die Verantwortung der Leiter des Staates nach seinem Rückkehr aus dem Exil im Sinne einer Aufhebung seines alten Gedankengutes neu zu definieren. ${ }^{41}$

\section{Wertbegriffe in Ciceros Sestiana}

Ciceros juristische Argumentation ist logisch und eindeutig. Wie könnte Sestius wegen vis verurteilt werden, wo er doch so lange das Wüten der clodianischen Horden erduldet hatte, und erst nachdem er auf dem Forum bei der Ausübung seines Amtes von der Bande des Clodius angegriffen wurde - und er es nur seinem Glück, bzw. jener irrigen Annahme der Attentäter, daß sie ihn für tot glaubten, sein Leben zu verdanken hat -, eine Leibstandarde zum eigenen Schutz aufstellte ${ }^{42}$ Sestius handelte in Notwehr, da ihm das Gesetz keinen

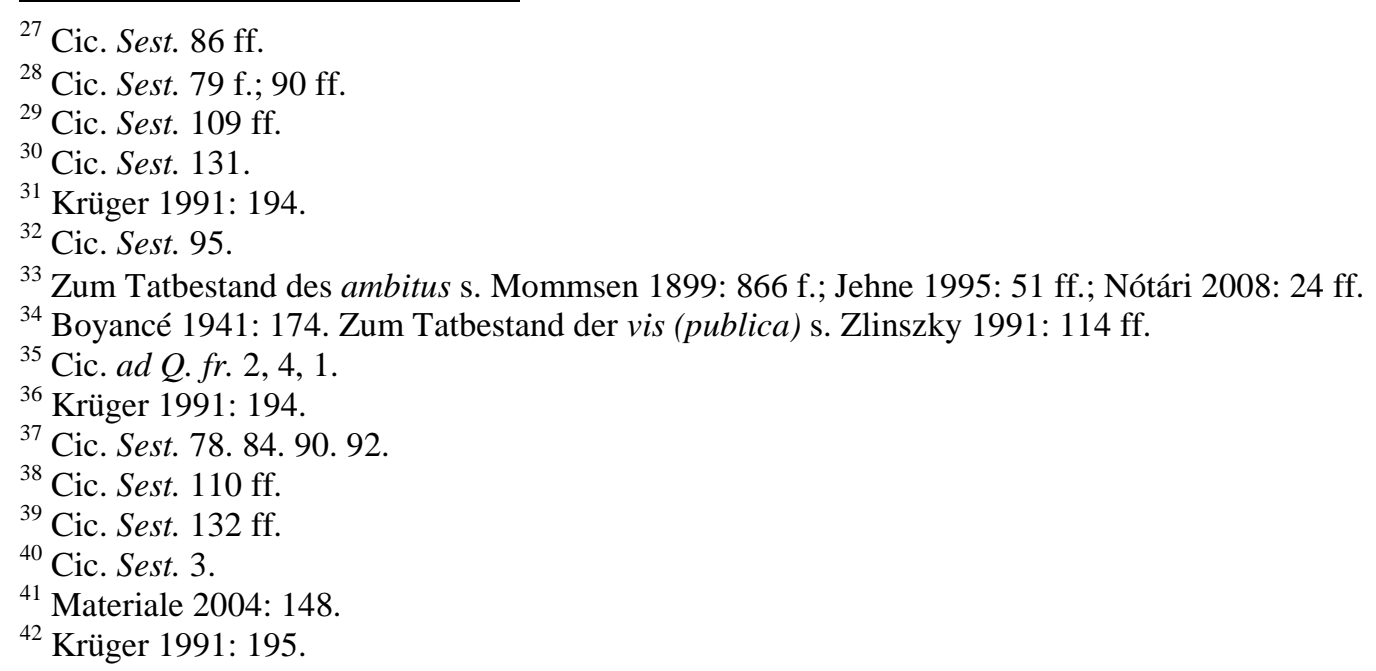


Schutz mehr zu geben vermöchte. ${ }^{43}$ Aufgrund dessen hat er sich keiner Straftat schuldig gemacht, ging aber vielmehr nach dem Prinzip des „vim vi”, bzw. „arma armis repellere cuique licet" vor. $^{44}$

Die Sestiana jedoch erscheint auf den ersten Blick als etwas unlogisch und unübersichtlich, die Rede selbst als überreich an Exkursen, besonders da sich mit der Person des Angeklagten nur relativ wenige Paragraphen befassen. ${ }^{45}$ Einen wesentlich größeren Raum nimmt der lebhafte Bericht von den Schicksalsschlägen und Triumphen, d.h. von dem Exil und Rückkehr des Redners selbst ein. ${ }^{46}$ Cicero führt seine Gedanken über das Gemeinwesen und die Rolle der leitenden Staatsmänner länger aus, die er der Jugend mit besonderem Nachdruck ans Herz legen möchte. ${ }^{47}$ Hierzu kommt das Prooemium ${ }^{48}$ und die Invektive gegen den Belastungszeugen Vatinius, der sich ironisch über die Optimaten geäußert, bzw. sie abfällig als natio optimatium ${ }^{49}$ bezeichnet hat. ${ }^{50}$ Oberflächlich betrachtet könnte man dazu neigen jener schon in den Antiken formulierten Ansicht beizupflichten, daß Cicero in seiner Rede viel zu weit vom eigentlichen Gegenstand des Prozesses abgewichen sei, bzw. daß die uns überlieferte Form der Pro Sestio mit der eigentlichen Gerichtsrede nicht viel zu tun hätte. ${ }^{51}$ Beim gründlicheren Lesen müssen wir allerdings die Meinung Manfred Fuhrmanns teilen, daß nämlich die Rede eine geschlossene, wohl durchdachte, logische Einheit bildet, da Cicero selber betont, daß - nachdem die einzelnen Anklagepunkte von Crassus, Hortensius und Calvus überzeugend widerlegt worden sind - er nichts anderes zu tun hätte, als den Lebenswandel und das Wirken des Sestius als Tribun im breit angelegten historischpolitischen Kontext zu würdigen. ${ }^{52}$ Somit läßt sich die Rede nach dem Prooemium gleichsam in einen historischen ${ }^{53}$ und einen programmatischen ${ }^{54}$ Teil untergliedern, die mit einem Fortissimo der peroratio schließt, in der Cicero pathetisch verkündet, daß falls der Angeklagte ins Exil gehen müßte, er selber keinen Augenblick zögerte dorthin Sestius, d.h. jenem Mann, dem er seinen Rückkehr zu verdanken hat, zu folgen. ${ }^{55}$

Es loht sich jenen Teil der Rede genauer zu untersuchen, die eventuell auch als bloßer und vielleicht sogar als unorganischer Exkurs abgestempelt werden könnte, allerdings aber gleichsam ein politisches Glaubensbekenntnis Ciceros über die Rolle und Verantwortung der Optimaten im Gemeinleben beinhaltet. ${ }^{56}$ Der paradigmatische Fall des Sestius bot dem Redner eine glänzende Gelegenheit seine staatsphilosophischen Gedankengänge vor großer Öffentlichkeit zu formulieren, die somit zu wesentlich mehreren Mitbürgern Zugang finden konnten, als wenn er sie in einem philosophisch-theoretischen Werk ausgeführt hätte. ${ }^{57}$ Was also auf den ersten Blick als bloßer Exkurs erscheinen mag, ist eine wohl durchdachte, überaus logisch und einprägsam aufgebaute Synthese: der Definition der Optimaten, die im abfälligen Ton natio optimatium genannt wurden, folgt eine Aufzählung der wichtigsten Aufgaben des Gemeinwesens, was in die Festlegung der für die Leiter der res publica als Pflicht aufgetragenen Grundwerte, des otium und der dignitas kulminiert.

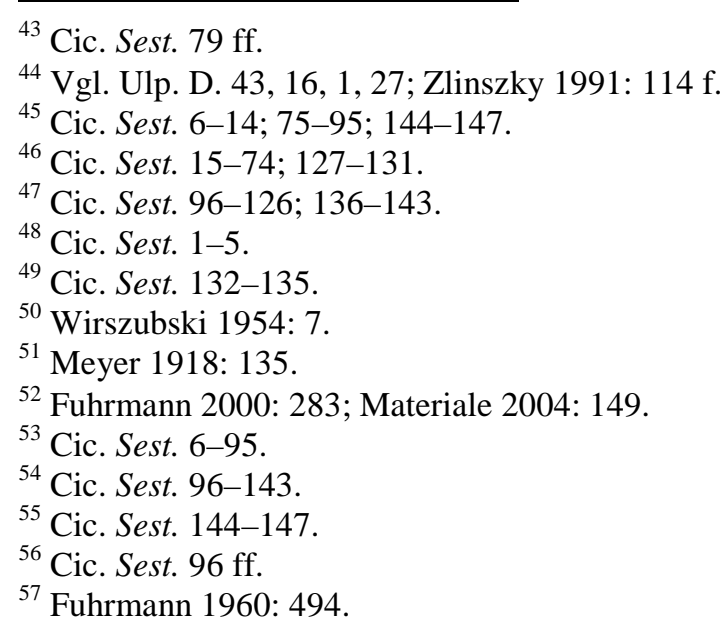


Als Antwort auf die dehonestierende Bemerkung des Anklägers formuliert Cicero mit Hilfe einer interpretatio extensiva seine eigene Optimatendefinition, und zwar indem er von dem „optimates-populares” Gegensatz ausgeht. Der Begriff der Optimaten oder der Popularen bedeutete in der politischen Terminologie der ciceronianischen Zeit natürlich weder eine Zugehörigkeit zu einer „Partei”, noch das Bekenntnis zu irgendwelchen politischen „Grundsätzen”, sondern bezeichnete in erster Linie die Gruppen jener Politiker, die bei der Verfolgung ihrer Ziele sich entweder auf den Senat, oder auf die Volksversammlung zu stützen versuchten, d.h. die Unterscheidung war eher stilistischer, als inhaltlicher Art. ${ }^{58}$ In seiner Sestiana macht Cicero den Versuch der bereits vorhandenen Form des Optimatenbegriffes einen prägnanteren Inhalt zu verleihen. Seiner Meinung nach versuchen die Optimaten - im Gegensatz zu den Popularen - nicht nach den Beifall der Menge zu erheischen, sondern sind bemüht die Anerkennung sämtlicher guter Bürger (optimus quisque) zu verdienen. ${ }^{59}$

Zur Gemeinschaft der optimi gehört jeder verantwortungsvoll denkender, unter ausgeglichenen Verhältnissen lebender Bürger, ungeachtet seines gesellschaftlichen Standes, d.h. selbst ein ,gutgesinnter" Freigelassener ist aus der Gruppe der optimi nicht ausgeschlossen. Ein „optimus quisque” ist also jeder anständiger römischer Bürger, angefangen von den Inhabern der höchsten Ämter und Mitglieder oberster Stände bis hin zu den römischen Stadtbewohnern, Bauern, Geschäftsleuten und Freigelassenen, d.h. alle, die nicht übelgesinnt oder aufrührerischer Natur sind, und sich nicht an Zwiespalt und Bürgerstreit erfreuen. Den Optimaten gegenüber - da der Begriff bis zu den äußersten Grenzen ausgedehnt wurde - stehen also nur die Krawallmacher und Aufrührer, die verwegenen Glücksritter der Politik, die Schädlinge des Gemeinwesens: „Quis ergo iste optimus quisque? Numero, si quaeris, innumerabiles, neque enim aliter stare possemus; sunt principes consili publici, sunt qui eorum sectam sequuntur, sunt maximorum ordinum homines, quibus patet curia, sunt municipales rusticique Romani, sunt negoti gerentes, sunt etiam libertini optimates. Numerus, ut dixi, huius generis late et varie diffusus est; sed genus universum, ut tollatur error, brevi circumscribi et definiri potest. Omnes optimates sunt qui neque nocentes sunt nec natura improbi nec furiosi nec malis domesticis impediti. esto igitur ut ii sint, quam tu 'nationem' appellasti, qui et integri sunt et sani et bene de rebus domesticis constituti. Horum qui voluntati, commodis, opinionibus in gubernanda re publica serviunt, defensores optimatium ipsique optimates gravissimi et clarissimi cives numerantur et principes civitatis. "60 Welches Ziel kann diese unzählige Arten von Menschen, diese riesige Gemeinschaft der Optimaten zusammenhalten und in Eintracht bringen, damit sie mit vereinten Kräften das Gemeinwohl erstreben? Der Redner hat die programmatische Antwort parat: „Quid est igitur propositum his rei publicae gubernatoribus quod intueri et quo cursum suum derigere debeant? Id quod est praestantissimum maximeque optabile omnibus sanis et bonis et beatis, cum dignitate otium." 61

Die politische Philosophie der Optimaten läßt sich nach Cicero mit zwei Grundwerten, bzw. deren Verknüpfung definieren: „cum dignitate otium”. ${ }^{2}$ Die dignitas ist immer eine Anerkennung, die jemandem entweder wegen einem persönlichen Verdienst, oder wegen seiner Abstammung zuteil wird. Es gilt daher zu betonen, daß die nicht mit der jedem zukommenden „Würde des Menschen” gleichzusetzen ist. Die dignitas ist in jedem Fall der Lohn eines im Gemeinleben erfüllten Amt, eines für das Gemeinwohl erwiesenen Dienstes, einer für die maiestas imperii/rei publicae aufgenommenen Bürde und Gefahr. Dignitas ist

\footnotetext{
${ }^{58}$ Krüger 1991: 196.

${ }^{59}$ Cic. Sest. 96. Vgl. Boyancé 1941: 179 ff.; Fuhrmann 1960: 484; Meyer 2005: 27 ff.

${ }^{60}$ Cic. Sest. 97.

${ }^{61}$ Cic. Sest. 98.

${ }^{62}$ Vgl. Rémy 1928: 113 ff. Zum otium s. ausführlicher Pérez 1995: 57 ff.
} 
also jener wohlverdiente Preis, der seinen Träger aus der grauen Masse der Durchschnittsmenschen hervorhebt. ${ }^{63}$ Sie ist jedoch nicht synonim mit honos oder laus, denn diese können durch das beispielhafte Meistern einer konkreten historisch-politischen Situation erlangt werden. Dignitas ist eine höhere, vor allem aber beständigere Kategorie: sie kommt gewissermaßen dem Begriff der nobilitas nahe, denn sie erstreckt sich weit über ein Amtsjahr oder einen Feldzug hinaus, kann von Generation zu Generation vererbt werden, und legitimiert somit den Einfluß und die Macht der späten Nachkommen auf das Gemeinwesen. Gerade in stürmischen Epochen des Gemeinwesens wird die ererbte dignitas von aufrührerischen Elementen angegriffen, daher gehört das Wahren dieses Grundwertes zu den eminentesten Aufgaben der Optimaten, jedoch nicht in ihrem eigenen Interesse, sondern vielmehr um dadurch das Gemeinwohl und die Stabilität des Staates zu hüten. ${ }^{64}$

Das otium ist gewissermaßen das Gegenteil des negotium, d.h. jede Tätigkeit außerhalb des öffentlichen Lebens. Es wird des öfteren mit den Begriffen pax, concordia, salus, quies und tranquillitas in Verbindung gebracht, und bildet gleichsam einen Gegensatz zu novae res, seditio, discordia und tumultus. Somit kann sowohl dignitas, wie auch otium als Eigen einer Einzelperson, ${ }^{65}$ einer Gruppe, ${ }^{66}$ einer Institution, bzw. eines Reiches oder Gemeinwesens angesehen werden, ${ }^{67}$ oder kann die öffentliche Ruhe, bzw. öffentliche Sicherheit bezeichnet werden. ${ }^{68}$

Es stellt sich auch die Frage, ob sich die Begriffe otium und dignitas auf Inhalte öffentlicher oder privater Natur beziehen. Rémy trennt an diesem Punkt sehr streng den auf das Kollektivum und den auf das Individuum bezogene Verwendung der besagten Grundbegriffe, ${ }^{69}$ und ist der Ansicht, das sie in der Sestiana die idealen, bzw. wünschenswerten Verhältnisse des öffentlichen Lebens spiegeln, d.h. daß die dignitas die Herrschaft des ordo senatorius und das otium die aus diesem Gleichgewicht entstammende öffentliche Ruhe bezeichnet. ${ }^{70}$ An anderen Stellen ist zu sehen, daß Cicero den Begriff der dignitas für die Bezeichnung des Einflusses einer Einzelperson, bzw. Senators, und otium als Synonim der ihm nach Niederlage seines Amtes zuteilgewordenen wohlverdienten Ruhe verwendet hat. ${ }^{71}$ Die Ansichten, mit der Rémy die Begriffe dignitas und otium strikt auf die öffentliche Sphäre beschränkte fanden in der Fachliteratur keine allgemeine Zustimmung. Boyancé betonte, daß in der Gedankenwelt Ciceros digintas in der Privatsphäre ebenso einen festen Platz hat, wie in der öffentlichen, und versuchte den ciceronianischen Begriff aus der griechischen, vorwiegend peripatetischen Philosophie abzuleiten. ${ }^{72}$ Wirszubski erachtete sowohl die Annäherung des Begriffes der dignitas an die Privatsphäre für etwas übertrieben, als auch ihre Ableitung aus der griechischen Philosophie für durchaus problematisch. Er selber aber mag sich wohl der Frage auch etwas einseitig angenommen haben, als er dignitas als eine ausschließlich politische, jeglicher philosophischen und ethischen Konnotation entbehrende Kategorie bewertete. ${ }^{73}$

Manfred Fuhrmann machte jene scharfsinnige und für seine synthetisierende Sichtweise durchaus nicht untypische Feststellung, daß sowohl Boyancés ausschließlich auf der griechischen Philosophie basierende, wie auch Wirszubskis außer der römischen Realpolitik

\footnotetext{
${ }^{63}$ Vgl. Wirszubski 1954: 12. So auch Büchner 1957: $322 \mathrm{ff}$.

${ }^{64}$ Fuhrmann 1960: $487 \mathrm{f}$.

${ }^{65}$ Cic. Sest. 125.128 f.; off. 3, 1.

${ }^{66}$ Cic. Sest. 104; fam. 1, 8, 4.

${ }^{67}$ Cic. Sest. 1.

${ }^{68}$ Cic. Sest. 15. 46. 104. Vgl. Fuhrmann 1960: 488 f.; Wirszubski 1954: 4 ff.

${ }^{69}$ Rémy 1928: 113 ff.

${ }^{70} \mathrm{Vgl}$. Heinze 1924: $73 \mathrm{ff}$.

${ }^{71}$ Fuhrmann 1960: 482.

72 Boyancé 1941: 186 ff.

${ }^{73}$ Wirszubski 1954: 3 ff.
} 
alle anderen Faktoren ignorierende Ansicht allzu einseitig, und daher unrichtig sei. Seines Erachtens dienten die Ergebnisse der griechischen Philosophie für Cicero als Mittel dazu seine eigenen Gedanken bezüglich des römischen Staatswesens zu artikulieren.

Cicero bezieht das Prinzip „cum dignitate otium” sowohl auf das Gemeinwesen im allgemeinen, als auch auf die Leiter des Staates im konkreten Sinne, aber gerade deswegen darf er in Anbetracht der Grundzüge römischen Staatsdenkens nicht einer wissentlich unklaren Verwendung der beiden Begriffe bezichtigt werden, wie es Wirszubski tat. ${ }^{74}$ Eben die ciceronianische Definition der res publica macht die Synthese der ,persönlichen” und „sachlichen” Komponente des Staatswesens, d.h. der abstrakten Staatsmacht und deren konkreten Ausübung durch die politische Elite geradezu erforderlich.

Der Zustand des otium läßt sich nach Ciceros Meinung nur dann verwirklichen, wenn die Leitung des Gemeinwesens in den Händen der Optimaten liegt, deren dignitas vom römischen Volk anerkannt wird, wodurch die optimi das otium und die dignitas der res publica, d.h. die Stabilität der Institutionen der Religion, der Rechtsprechung, der Finanzen, der Innen- und Außenpolitik, der Zivil- und Militärverwaltung garantieren können: „Huius autem otiosae dignitatis haec fundamenta sunt, haec membra, quae tuenda principibus et vel capitis periculo defendenda sunt: religiones, auspicia, potestates magistratuum, senatus auctoritas, leges, mos maiorum, iudicia, iuris dictio, fides, provinciae, socii, imperi laus, res militaris, aerarium. "75

Dieses fragile Gleichgewicht wird sogar aus zwei Richtungen gefährdet. Einerseits machen es clodiusartige aufrührerische Krawallmacher und verwegene politische Anarchisten, ${ }^{76}$ andererseits jene Bürger unsicher, die von den zwei organisch zusammenhängenden Grundwerten nur das eine oder das andere im Auge zu behalten fähig sind, und entweder nach dignitas streben ohne auf das otium zu achten, oder aber sich bereit zeigen auf die dignitas zu verzichten, um otium wahren zu können. Den letzteren, d.h. den zögernden und trägen wird eben jene Gefahr nicht bewußt, daß indem sie nach der trügerischen Illusion der Sicherheit trachten mit dem Verzicht auf die dignitas auch das otium leicht verlorengehen kann: „Maioribus praesidiis et copiis oppugnatur res publica quam defenditur, propterea quod audaces homines et perditi nutu impelluntur et ipsi etiam sponte sua contra rem publicam incitantur, boni nescio quo modo tardiores sunt et principiis rerum neglectis ad extremum ipsa denique necessitate excitantur, ita ut non numquam cunctatione ac tarditate, dum otium volunt etiam sine dignitate retinere, ipsi utrumque amittant."

Im weiteren zählt Cicero eine Reihe jener propugnatores rei publicae auf, die der Mühe und Arbeit nicht scheuten und das Gemeinwesen vor dem aufrührerischen Treiben der Popularen retteten, was zu jenen Zeiten mit erheblich größeren Gefahren verbunden war, da damals die Politik der Popularen beim Volk Gefallen gefunden hatte. ${ }^{78}$ Für seine Zeit stellt der Redner allerdings fest, daß die Bestrebungen der Popularen beim verus populus, ${ }^{79}$ d.h. beim verantwortlich denkenden, die Richtigkeit der Politik der Optimaten gutheißenden und sich nach otium sehnenden Volk auf Abneigung stoßen, und daß clodiusartige Anarchisten nur noch beim aufgehetzten oder erkauften Pöbel Beifall finden können: „Nunc iam nihil est quod populus a delectis principibusque dissentiat: nec flagitat rem ullam neque novarum rerum est cupidus et otio suo et dignitate optimi cuiusque et universae rei publicae gloria delectatur. Itaque homines seditiosi ac turbulenti, quia nulla iam largitione populum

\footnotetext{
${ }^{74}$ Vgl. Wirszubski 1954: 7 ff.

${ }^{75}$ Cic. Sest. 98. Vgl. Krüger 1991: 197 f.; Fuhrmann 2000: 285; Materiale 2004: 151; Meyer 2005: 38 ff.

76 „Anarchist” ist im Fall des Clodius zwar eine etwas anachronistische, jedoch durchaus zutreffende Bezeichnung. So auch Alföldi 1985: 128.

${ }^{77}$ Cic. Sest. 100. Vgl. Boyancé 1941: 184 ff.

${ }^{78}$ Cic. Sest. 101 ff. Vgl. Fuhrmann 1960: 485.

${ }^{79}$ Cic. Sest. 108.114.
} 
Romanum concitare possunt, quod plebes perfuncta gravissimis seditionibus ac discordiis otium amplexatur, conductas habent contiones, neque id agunt ut ea dicant aut ferant quae illi velint audire qui in contione sunt, sed pretio ac mercede perficiunt ut, quicquid dicant, id illi velle audire videantur."

Er fordert die römischen Bürger auf, die dieser ausgedehnten Definition folgend von den feindlich gesinnten Elementen abgesehen allesamt zu den Optimaten zählt, dem Beispiel der eben erwähnten, nach Ruhm, Ansehen und Anerkennung strebenden Männer nachzueifern, verschweigt jedoch nicht, daß diese Aufgabe mühevoll und mit vielen Gefahren verbunden ist: „Haec imitamini, per deos immortalis, qui dignitatem, qui laudem, qui gloriam quaeritis! Haec ampla sunt, haec divina, haec immortalia; haec fama celebrantur, monumentis annalium mandantur, posteritati propagantur. Est labor, non nego; pericula magna, fateor." 81

Die Anführer der Optimaten, die principes civitatis, die dem um das Gemeinwohl, die Freiheit, die öffentliche Ruhe und die Würde des Volkes sorgenden Senat folgen, ihren Feinden, den audaces und den improbi, die nicht selten aus dem Kreis der potentes stammen, mutig ins Auge sehen müssen, die Exempel aus der Geschichte zeigen jedoch, daß diese Aufrührer und Unruhestifter, die nach dem Beifall des Pöbels trachten, größtenteils ein elendes Ende finden. ${ }^{82}$

An dieser Stelle ermahnt Cicero die Jugend, daß sie - denn eben diesem Zweck diente der lange Exkurs über die Definition des Optimatenbegriffes ${ }^{83}$ - jene dignitas und gloria vor Augen halten sollte, welche durch die Verdienste um die res publica erworben werden können, er fürchte nämlich, daß wegen dem Wirren der letzten Jahre und den ihn treffenden Schicksalsschlägen alle boni davor zurückschreckten die Bürde und Verantwortung der Staatsgeschäfte auf sich zu nehmen. ${ }^{84}$ Daher betont er mit Nachdruck, daß er zwar - wie zahlreiche Vorkämpfer der Opimatenpolitik - verbannt, aber sehr bald heimgerufen und in seine alte dignitas eingesetzt worden ist. ${ }^{85}$

\section{Konklusionen}

Die Analyse der Rolle der Optimaten im öffentlichen Leben überschritt weit das für die Sache des Sestius erforderliche Maß, stand aber - wie von Cicero selber betont - im organischen Zusammenhang mit den anderen Elementen seiner Verteidigungsrede. ${ }^{86}$ Trotz der rhetorischen Übertreibungen müssen wir in diesem Punkt Cicero beipflichten. ${ }^{87}$ Sestius stand zweifelsohne auf der Seite der Optimaten, d.h. auf der Seite eines jeden guten Bürgers (quisque optimus), wie er es mit seinem Lebenswandel und seinem politischen Handeln bewiesen hatte, ${ }^{88}$ denn er ergriff nicht nur neben dem für das Gemeinwesen heroische Aufgaben meisternde Cicero Partei, sondern vertrat auch die Interessen des Senats, Italiens und der ganzen res publica, ${ }^{89}$ indem er sich den fanatischen Aufrührern, Hochverrätern und politischen Glücksrittern, von denen der Redner ein mit vernichtendem Sarkasmus gezeichnetes Bild gibt, mutig gestellt hatte. ${ }^{90}$ Es ist zwar fraglich, ob die von Gabinius und

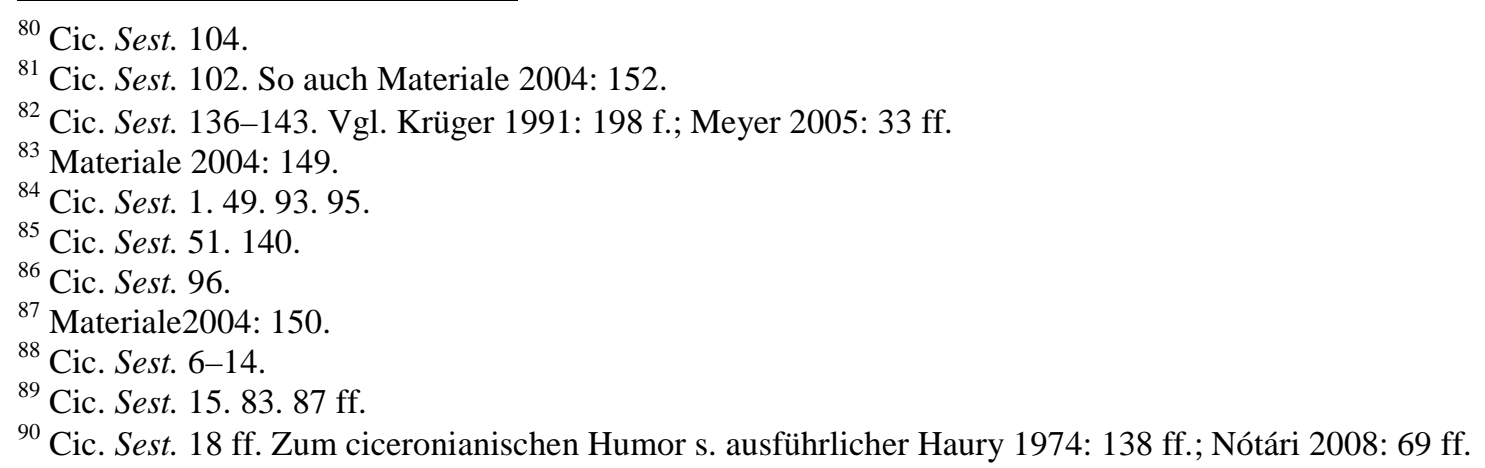


Piso gezeichneten, an manchen Punkten in Karikaturen übergehenden Portraits in allen Deitails der historischen Wirklichkeit entsprechen, aber ihrem literarischen Wert nach sind sie Meisterstücke ciceronianischer Redekunst. Im Kampf, für den sich Milo, Sestius, der Senat, die Bürgerschaft und ganz Italien ${ }^{91}$ gegen den Staat ins Verderben stürzenden Clodius, Gabinius, Piso und das aufgehetzte Pöbel ${ }^{92}$ verbündeten, stand nicht nur die Rückberufung Ciceros aus dem Exil auf dem Spiel, sondern vielmehr die otiosa dignitas, ${ }^{93}$ d.h. Existenz der Grundwerte des Gemeinwesens, welche die ihnen anvertraute Macht mißbrauchenden Anarchisten vernichten wollten. ${ }^{94}$

Der einprägsamen Ausführung des Prinzips „cum dignitate otium”, das Cicero wohl neben der Erschaffung des consensus, bzw. der concordia ordinum schon zu seiner Konsulatszeit vorgeschwebt haben muß, ${ }^{95}$ blieb auch der Erfolg im Prozeß gegen Sestius nicht versagt, denn der Angeklagte wurde einstimmig freigesprochen - ein Ergebnis, dem Cicero, wie es ein Brief an seinen Bruder zeigt, höchste politische Wichtigkeit beimaß. ${ }^{96}$ Die Sestiana gab dem Redner eine willkommene Gelegenheit mit seinem staatsphilosophisch-politischen Programm in Form einer Gerichtsrede vor die Öffentlichkeit zu treten, denn es stellte sich für einen kurzen Augenblick in Rom ein verhältnismäßiges Gleichgewicht der einander widerstrebenden Kräften ein, was auch jenem Umstand zu verdanken war, daß sich Caesar fern von Rom aufhielt, bzw. nicht direkt ins Geschehen eingreifen wollte.

Leider fehlte es zur Verwirklichung der hier definierten Ziele auch Cicero an Mitteln, denn Rom wurde nunmehr zu jenem Zentrum, wo - wie es das Beispiel Caesars überdeutlich machte - politische Entscheidungen höchstens legitimiert, nicht aber getroffen werden sollten. Caesar traf bald mit Crassus und Pompeius zusammen und erneuerte das Bündnis im Sinne des ersten Triumvirats. Für eine kurze Weile blieb noch das otium erhalten, die dignitas ging allerdings verloren. Die Politik der Popularen, der sich Caesar an die Spitze stellte, fand nicht nur bei dem Pöbel und den anarchistisch gesinnten Elementen der Stadt und des Reiches Beifall, sondern auch bei manchen, die Cicero in seiner Rede für Sestius zu den Optimaten zählen zu dürfen gehofft hatte. ${ }^{97}$

In der Pro Sestio gab Cicero allerdings ein geniales Beispiel dafür, wie staatsphilosophisches Gedankengut in Form einer Gerichtsrede als programmatischer Aufruf zur Besinnung auf Grundwerte und Prinzipien menschlichen Zusammenlebens eindrucksvoll vermittelt werden kann. Der glückliche Einklang zwischen dignitas und otium, d.h. den idealistischen Grundwerten und materiellen Interessen, bzw. die Aufforderung zur manchmal sogar mühsamen und Opfer verlangenden Verwirklichung dieses Einklanges sichern der Sestiana einen festen Platz unter den Bravurreden Ciceros, und machen sie zugleich zu einem der wichtigsten Vorlagen des in seiner Schrift De re publica herausgearbeiteten staatsphilosophischen Gedankengutes.

Rednerische Brillianz, aktualpolitisches Programm und staatsphilosophische Weitsicht bilden eine harmonische Synthese in Ciceros Verteidigungsrede für Sestius. In der Sestiana gab Cicero für die verantwortungsvoll denkende Elite und Bürgerschaft Roms eine Wegweisung zur Aufrechterhaltung, bzw. Widerherstellung der Stabilität der res publica und eine Ermahnung zur Neudefinition jener klassischer Wertbegriffe, die detaillierter ausgearbeitet und in aller Reife im Werk De re publica wiederaufgefunden werden können.

\footnotetext{
${ }^{91}$ Cic. Sest. 32. 36. 53. 72.

${ }^{92}$ Cic. Sest. 25.

${ }^{93}$ Cic. Sest. 98.

${ }^{94}$ Cic. Sest. 25 ff.; 32 ff.; 42 ff.; 55 f.; 66.75 ff.; 89 ff.; 93 ff.

${ }^{95}$ Materiale 2004: 147; Wirszubski 1954: 8. Zum Begriff der concordia ordinum s. Strasburger 1931.

${ }^{96}$ Cic. $a d$ Q. fr. $2,4,1$.

${ }^{97}$ Fuhrmann 2000: 288.
} 
Alföldi, Andreas (1985): Caesar in 44 v. Chr. I. Studien zu Caesars Monarchie und ihren Wurzeln, Bonn: Habelt. Bleicken, Jochen (1959): Ursprung und Bedeutung der Provocation, in: Zeitschrift der Savigny-Stiftung für Rechtsgeschichte, 76. Jg., S. 324-377.

Bleicken, Jochen (1975): Die Verfassung der römischen Republik, Paderborn: Schöningh.

Boyancé, Pierre (1941): Cum dignitate otium, in: Revue des Études Anciennes, 43. Jg., S. 172-191.

Büchner, Karl (1957): Humanitas Romana, Heidelberg: Winter.

Classen, Carl Joachim (1973): Ciceros Rede für Caelius, in: Temporini, Hildegard (Hrsg.): Aufstieg und Niedergang der römischen Welt, Bd. I/3, Berlin-New York: de Gruyter, S. 60-94.

Drexler, Hans (1976) Die Catilinarische Verschwörung, Darmstadt: Wissenschaftliche Buchgesellschaft.

Epstein, David F. (1986): Cicero's testimony at the Bona Dea Trial, in: Classical Philology, 80. Jg., S. $229-235$.

Fuhrmann, Manfred (1960): Cum dignitate otium. Politisches Programm und Staatstheorie bei Cicero, in: Gymnasium, 67. Jg., S. 481-500.

Fuhrmann, Manfred (2000): Rede für Sestius, in: Fuhrmann, Manfred (Hrsg.): Marcus Tullius Cicero, Sämtliche Reden, Bd. 5, Düsseldorf-Zürich: Artemis. S. 281-285.

Geffcken, Katherine A. (1973) Comedy in the pro Caelio, Leiden: Brill.

Giebel, Marion (1977): Marcus Tullius Cicero, Reinbek bei Hamburg: Rowolt.

Gruen, Erich S. (1966): P. Clodius: Instrument or Independent Agent?, in: The Phoenix, 20. Jg., S. 120-130.

Haury, Auguste (1955): L'ironie et l'humour chez Ciceron, Paris: Les belles letters.

Heinze, Richard (1924): Ciceros Staat als politische Tendenzschrift, in: Hermes, 59. Jg., S. 73-94.

Heinze, Richard (1925): Ciceros Rede pro Caelio, in: Hermes, 60. Jg., S. 193-258.

Jehne, Martin (1995): Die Beeinflussung von Entscheidungen durch „Bestechung”: Zur Funktion des ambitus in der römischen Republik, in: Jehne, Martin (Hrsg.): Demokratie in Rom? Die Rolle des Volkes in der Politik der römischen Republik, Stuttgart: Steiner, S. 51-76.

Köves-Zulauf, Thomas (1995): Bevezetés a római vallás és monda történetébe (Einführung in die Geschichte der römischen Religion und Sage), Budapest: Telosz.

Krüger, Gerhard (1991): M. Tullius Cicero, Pro P. Sestio oratio, Stuttgart: Reclam.

Kunkel, Wolfgang (1962): Untersuchungen zur Entwicklung des römischen Kriminalverfahrens in vorsullanischer Zeit, München: Beck.

Lintott, Andrew W. (1974) Provocatio. From the Struggle of the Orders to the Principate, in: Temporini, Hildegard (Hrsg.): Aufstieg und Niedergang der römischen Welt, Bd. I/2, Berlin-New York: de Gruyter, S. 226277

Martin, Jochen (1970): Die Provokation in der klassischen und späten Republik, in: Hermes, 98. Jg., S. $72-96$.

Martin, Josef (1974): Antike Rhetorik. Technik und Methode, München: Beck.

Materiale, Filippo (2004): L'ideale politico di Cicerone nella pro Sestio, in: Salerno, Franceso (ed.): Cicerone e la politica, Napoli: Satura, S. 145-152.

Meier, Christian (1968) Ciceros Consulat, in: Radke, Gerhard (Hrsg.): Cicero, ein Mensch seiner Zeit, Berlin: de Gruyter, S. 61-116.

Meyer, Eduard (1918): Caesars Monarchie und das Prinzipat des Pompeius, Stuttgart-Berlin, Klett.

Meyer, Inga (2005): Von der Vision zur Reform. Der Staat der Gesetze - Ciceros Programm einer Neuordnung der Römischen Republik: 56-51 v. Chr., München: Utz.

Mommsen, Theodor (1899): Römisches Strafrecht, Leipzig: Duncker \& Humblot.

Moreau, Philippe (1982): Clodiana religio. Un procès politique en 61 av. J.-C., Paris: Les belles lettres.

Münzer, Friedrich (1920): Römische Adelsparteien und Adelsfamilien, Stuttgart: Metzler.

Nótári, Tamás (2008): Law, Religion and Rhetoric in Cicero's Pro Murena, Passau: Schenk.

Perelli, Luciano (1990): Il pensiero politico di Cicerone, Firenze: Nouva Italia.

Pérez, José Joaquín Caerols (1995): Arceri oti finibus (Cic. har. 4): ¿„paz” civil u „ocio” de lós jóvenes aristócratas?, in: Estudios Clásicos, 108. Jg., S. (1995), 57-92.

Rémy, Eduard (1928): Dignitas cum otio, in: Musée Belge, 32. Jg., S. 113-127.

Rundell, W. M. F. (1979): Cicero and Clodius: The Question of Credibility, in: Historia, 28. Jg., H. 3, S. 301328.

Salzman, Michele R. (1982): Cicero, the Megalenses and the defense of Caelius, in: American Journal of Philology, 103. Jg., S. 299-304.

Spielvogel, Jörg P. (1997): Clodius P. Pulcher - eine politische Ausnahmeerscheinung der späten Republik?, in: Hermes, 125. Jg., S. 56-74.

Strasburger, Hermann (1931): Concordia ordinum. Eine Untersuchung zur Politik Ciceros, Borne-Leipzig: Noske.

Stroh, Wilfried (1975): Taxis und Taktik. Die advokatische Dispositionskunst in Ciceros Gerichtsreden, Stuttgart: Teubner.

Süß, Wihelm (1965) Cicero. Eine Einführung in seine philosophische Schriften, Wiesbaden: Steiner. 
Uttschenko, Sergej Lvovič (1978): Cicero, Berlin: Deutscher Verlag der Wissenschaften.

Wirszubski, Chaim (1950): Libertas as a Political Idea at Rome during the Late Republic and Early Principate, Cambridge: Cambridge University Press

Wirszubski, Chaim (1954): Cicero's cum dignitate otium - A Reconsideration, in: Journal of Roman Studies, 44. Jg., S. 1-13.

Zlinszky, János (1991): Római büntetőjog (Römisches Strafrecht), Budapest: Tankönykiadó. 